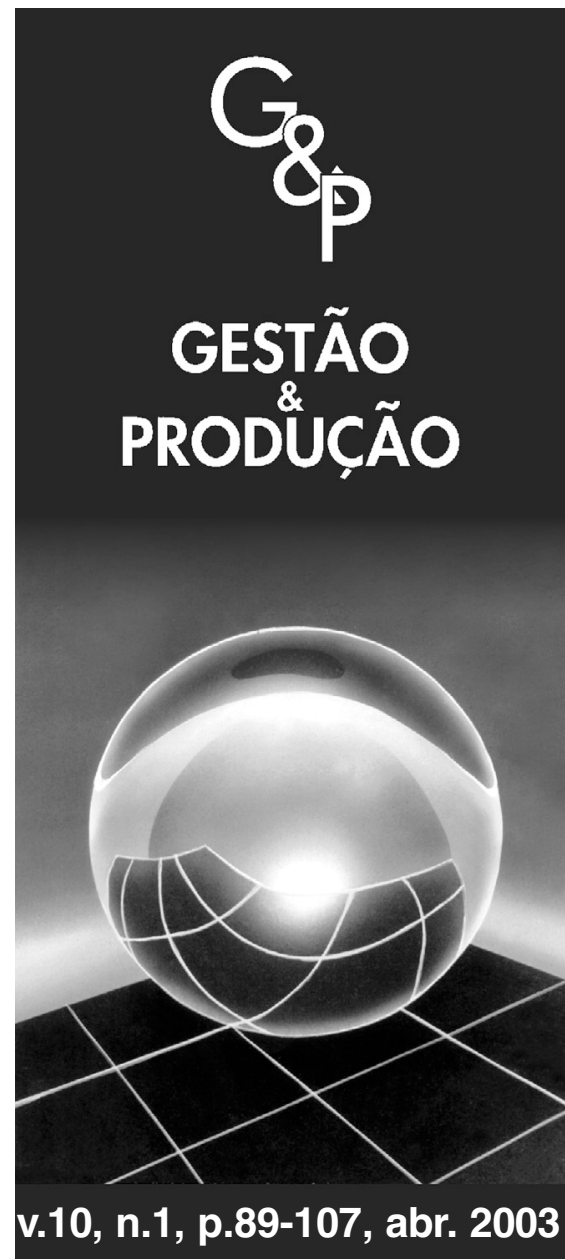

\title{
UM SISTEMA PARA CLASSIFICAR E CODIFICAR OS TRABALHOS QUE RELACIONAM O CONTROLE DA PRODUÇÃO E O CONTROLE DA QUALIDADE
}

\section{Moacir Godinho Filho Flavio César Faria Fernandes}

Departamento de Engenharia de Produção, Universidade Federal de São Carlos,

Via Washington Luís, km 235, CEP 13565-905, São Carlos, SP, e-mails: moacir_godinho@uol.com.br e dfcf@power.ufscar.br

\section{Resumo}

Este artigo apresenta um sistema para classificar e codificar trabalhos (SCCA - sistema de classificação e codificação de artigos) que relacionam o Controle da Produção $(C P)$ e o Controle da Qualidade (CQ). O sistema de classificação é baseado em três características principais do artigo: a natureza, o foco e os objetivos. O sistema de codificação emprega a idéia básica da tecnologia de grupo (codificação de famílias de peças), utilizando uma estrutura híbrida de três dígitos para codificar as características mencionadas anteriormente. O SCCA foi criado em função da realização de um survey, o qual procurou ser o mais completo possível, contendo 45 trabalhos que relacionam o $C P$ e o $C Q$. $O$ sistema é validado por sua utilização na classificação e codificação dos trabalhos encontrados no survey. Essa classificação e codificação serve, ao mesmo tempo, como auxílio para estruturar a revisão bibliográfica e para realizar uma análise geral sobre o tema. A partir dessa análise, este artigo consegue proporcionar maior conhecimento e comunicação das pesquisas existentes atualmente que relacionam o CP e o CQ, além de sugerir novas áreas de pesquisa dentro das relações entre CP e CQ. $O$ artigo destaca ainda a importância de estudar a relação entre $C P$ e $C Q$.

Palavras-chave: controle da produção, controle da qualidade, sistema de classificação e codificação, revisão bibliográfica. 


\section{Introdução}

$\mathrm{N}$ este trabalho apresentamos um sistema de classificação e codificação de artigos (SCCA) existentes atualmente na área acadêmica, os quais relacionam o Controle da Produção $(\mathrm{CP})$ e o Controle da Qualidade (CQ). O interesse em estudar as relações entre $\mathrm{CP}$ e $\mathrm{CQ}$ reside no fato de acreditarmos que a multiplicidade de objetivos estratégicos da produção é uma realidade que as empresas vêm enfrentando no mundo atual, altamente competitivo. Nas palavras de Sipper \& Bulfin (1997): “(...) nos tempos atuais os objetivos são múltiplos para as empresas. Elas devem: produzir bens e serviços de qualidade soberba, entregar exatamente no momento que o cliente deseja, a um mínimo custo possível e que ainda forneça um retorno satisfatório para os acionistas (...)". Essa visão holística foi desenvolvida por Fernandes \& Maccarthy (1999) em artigo recente e está sumarizada na Figura 1. À medida que se vai da manufatura repetitiva para a manufatura ágil, novos objetivos vão sendo levados em conta, sem desprezar os objetivos já incorporados. Por exemplo, enquanto a manufatura repetitiva foca a questão do aumento da produtividade/redução de custo, a manufatura enxuta foca a produtividade/custo e a qualidade.

Podemos ver na Figura 1 que a manufatura enxuta (Sistema Toyota de Produção) engloba os objetivos qualidade (relacionado diretamente ao CQ) e produtividade/custo (relacionado diretamente ao $\mathrm{CP}$ ).

Portanto, podemos dizer que estudar as relações entre CP e CQ é contribuir para alcançar a multiplicidade de objetivos no âmbito da manufatura enxuta, além de estimular a integração entre os processos, em prol de aumento da competitividade das empresas. Pois, de acordo com Rummler \& Brache (1994), é na identificação e na eliminação dos "fios desligados" (relacionamentos falhos entre processos e funções de uma organização) que se encontram, com freqüência, as maiores oportunidades para o aperfeiçoamento do desempenho da organização e, conseqüentemente, para o aumento de sua competitividade.

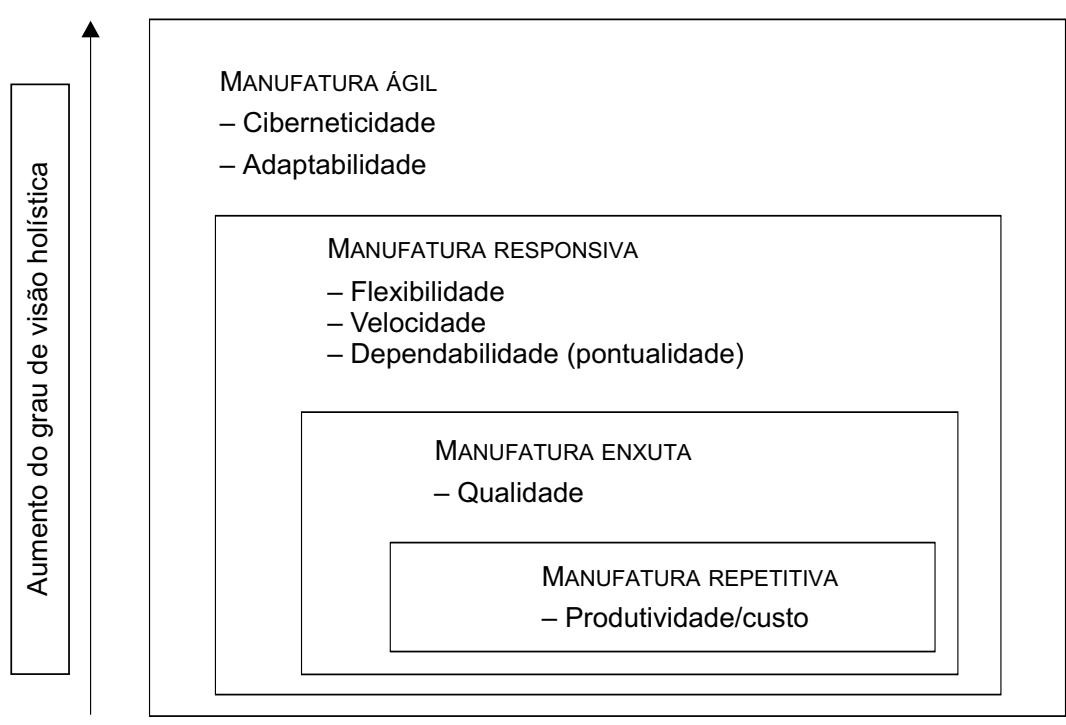

Figura 1 - Relação entre as manufaturas repetitiva, enxuta, responsiva e ágil (Fernandes \& MacCarthy, 1999). 
O conhecimento científico se baseia em classificações. Portanto, a classificação é ferramenta essencial para o conhecimento de determinada área. $\mathrm{Na}$ área das relações entre $\mathrm{CP}$ e CQ não é diferente.

De acordo com Good (1965), as classificações podem servir aos seguintes propósitos:

1. conhecimento mental e comunicação;

2. descobrimento de novo campo de pesquisa;

3. planejamento de estrutura organizacional ou estrutura de uma máquina;

4. como lista de conferência;

5. entretenimento.

A classificação a ser apresentada neste trabalho se insere, principalmente, no grupo (1) e também no grupo (2). Além desses objetivos, o sistema de classificação e codificação de artigos também servirá para estruturar a própria revisão bibliográfica sobre o tema. A estrutura da revisão que propomos, baseada em um sistema formal de classificação e codificação, pode ser usada como modelo para realizar outras revisões bibliográficas sobre outros temas. A revisão bibliográfica deste artigo representa uma contribuição, pois nenhuma outra possui os mesmos objetivos e características. Murthy \& Ma (1991) revisam os trabalhos que tratam dos sistemas MRP com incertezas devidas à qualidade. No artigo de Yano \& Lee (1995) é apresentada uma revisão da literatura de abordagens quantitativas para determinação do tamanho de lote quando o rendimento da produção ou a compra é aleatória; esse artigo concentra-se nas descrições dos tipos de problemas que foram resolvidos e em importantes resultados estruturais.

Antes de passarmos à classificação propriamente dita, é interessante definirmos os termos Controle da Produção (CP) e Controle da Qualidade (CQ), a fim de evitar confusões conceituais.

Para Sipper \& Bulfin (1997), o Planejamento e Controle da Produção (PCP) combina o fluxo físico e de informações com a finalidade de gerenciar os sistemas de produção.

Para Fernandes (1991), há muita confusão entre as atividades de Planejamento da Produção (PP) e de Controle da Produção (CP). De acordo com esse autor, tanto para o PP como para o $\mathrm{CP}$, as atividades de planejar e controlar são essenciais, porém o objetivo do PP é tratar as questões de médio prazo, como: planejar a capacidade produtiva para tentar compatibilizá-la com o comportamento esperado da demanda futura, fornecer diretrizes e parâmetros para que os contratos de fornecimento de médio prazo possam ser firmados, estabelecer políticas de subcontratação e terceirização, dentre outros. Já o CP visa regular, no curto prazo (até três meses), o fluxo de materiais por meio do fluxo de informações. A amplitude dos horizontes colocada não é rígida, já que, quanto maior a instabilidade, menor deve ser o horizonte de planejamento do CP.

Para Burbidge (1990), o CP é função do gerenciamento que planeja, dirige e controla a cadeia de materiais e as atividades de processo de produto em uma empresa ou, em outras palavras, é a função de gerenciamento que controla o fluxo de materiais nos centros de trabalho.

Entendemos CP conforme os autores vistos anteriormente, ou seja, como a função do gerenciamento que regula (planeja, coordena, dirige e controla), no curto prazo, o fluxo de materiais e as atividades em um sistema de produção por meio do fluxo de informações e decisões.

Já por CQ adotaremos o ciclo de Controle de Deming, conhecido como ciclo PDCA (Plan-DoCheck-Act), o qual Hicks (1994) divide em:

1. determinar objetivos e metas;

2. determinar métodos para atingir $\} P$ essas metas;

3. treinamento;

4. implementação do trabalho; $\}$ D

5. checar os efeitos da implementação; \}C

6. tomar medidas apropriadas. $\}$ A 
Tanto a estrutura do CP quanto a do CQ são compatíveis com a definição de Taylor para controle, ou seja, significa plan-do- "see" (Ishikawa, 1981).

O artigo apresenta a seguinte estrutura: na Seção 2 apresentamos a metodologia utilizada; na Seção 3, o SCCA é proposto e utilizado na classificação e na codificação dos artigos do survey; na Seção 4 utilizamos o SCCA para estruturar a revisão bibliográfica dos artigos do survey; na Seção 5, realizamos uma análise geral dos 45 trabalhos encontrados no survey, à luz do sistema proposto e, finalmente; na Seção 6, tecemos algumas conclusões, nas quais incluímos sugestões para futuros trabalhos, relacionando $\mathrm{CP}$ e CQ, que tratem aspectos pouco explorados, conforme evidenciado por nosso sistema de classificação e codificação.

\section{Metodologia}

Este trabalho foca a elaboração do SCCA sobre as relações entre $\mathrm{CP}$ e $\mathrm{CQ}$. Tal sistema foi proposto (Seção 3) em função da realização de um survey (45 artigos), o qual procurou ser o mais completo possível, sobre as relações entre CP e CQ. Uma vez proposto o SCCA, este foi validado por sua aplicação na classificação e codificação de artigos do survey (Seção 3). Com base nisto, pôde-se estruturar a revisão bibliográfica de forma mais conveniente (Seção 4) e também realizar a análise geral do tema proposto (Seção 5). Essa análise possibilita melhor conhecimento do tema e sugere futuras pesquisas dentro dele (Seções 5 e 6 ).

A Figura 2 mostra a metodologia de estruturação deste trabalho, salientando os pontos que serão discutidos nas próximas seções.

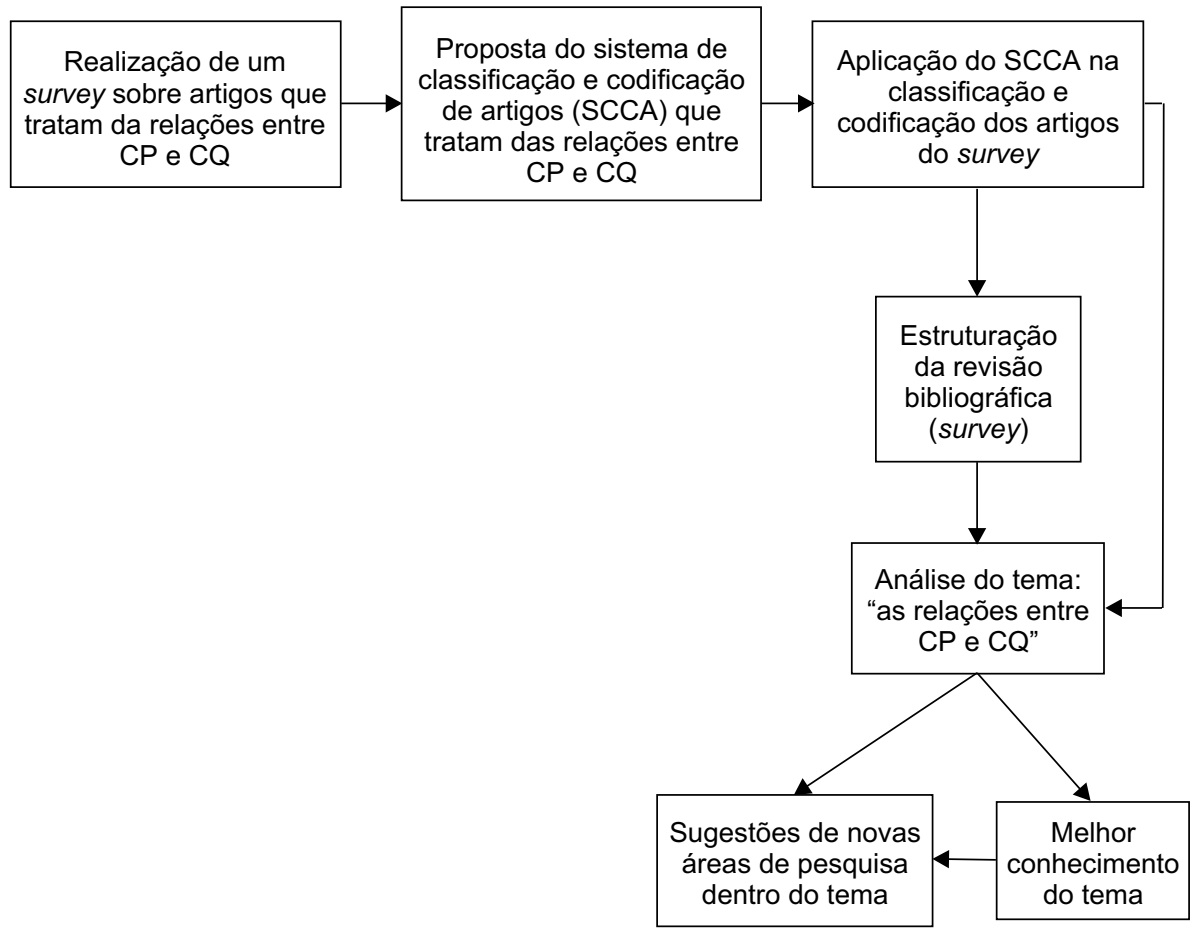

Figura 2 - Metodologia e estruturação do trabalho. 


\section{Proposta e aplicação de um sistema de classificação e codificação de trabalhos que relacionem o CP com o CQ}

Propomos neste trabalho um sistema de classificação e codificação, o qual pode ser estruturado após um estudo detalhado dos 45 trabalhos encontrados no survey entre as relações entre $\mathrm{CP}$ e CQ. Tal sistema de classificação é baseado em três características principais:

- natureza do artigo;

- foco do artigo;

- objetivos do artigo.

Juntamente com a classificação é proposto um sistema de codificação. Para melhor entendimento de nosso sistema de codificação, é importante descrever algumas estruturas básicas usadas em trabalhos de codificação relacionados à tecnologia de grupo (codificação de famílias de peças). De acordo com Groover \& Zimmers (1984), estas estruturas são:

- Estrutura hierárquica - na qual a interpretação de cada código depende do valor do código anterior. Também é chamada de estrutura em árvore ou monocode.

- Estrutura em cadeia - na qual a interpretação de cada símbolo na sequiência é fixa e não depende do valor dos dígitos precedentes. Também é chamada de estrutura policode.
- Estrutura híbrida - é uma combinação das duas estruturas puras mostradas anteriormente. A maioria dos sistemas comerciais de codificação de peças utiliza estrutura híbrida. Um dos mais conhecidos dentre esses sistemas é o famoso sistema Opitz (Opitz et al., 1969).

Em nosso sistema de codificação utilizaremos a estrutura híbrida, sendo que as características natureza do artigo e objetivos do artigo apresentam estrutura em cadeia, enquanto a característica foco do artigo tem estrutura hierárquica, ou seja, sua codificação dependerá do atributo do código anterior (relativo à característica natureza do artigo).

A Figura 3 ilustra como será nosso sistema de codificação. Vemos que o primeiro dígito corresponde à característica natureza do artigo, o segundo dígito, à característica foco do artigo, enquanto os dígitos seguintes são relativos aos objetivos do artigo. As duas primeiras características serão representadas por apenas um dígito cada, enquanto a última poderá ser representada por diversos dígitos, correspondentes aos objetivos, lembrando que para cada objetivo haverá um código de dois dígitos correspondente, sendo esses códigos de objetivos separados por um traço (-). Além desses códigos, uma informação importante que aparece em nosso sistema de classificação e codificação é a data do periódico (logo após a característica natureza do artigo, entre parênteses).

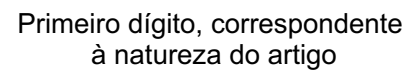

Segundo dígito, correspondente ao foco do artigo (depende do $1^{\circ}$ dígito)
Terceiro dígito, correspondente aos objetivos do artigo

Figura 3 - Esquema geral de codificação. 
Após essas explicações, passaremos às classes existentes dentro de nossas três características.

A característica natureza do artigo é composta por duas grandes classes:

- Trabalhos quantitativos - (representaremos esta classe pela letra Q) dentro desta classe estão os trabalhos que utilizam métodos quantitativos (fórmulas matemáticas, modelos, etc.) para moldar ou explicar a relação entre $\mathrm{CP}$ e CQ.

- Trabalhos qualitativos - (representaremos esta classe pela letra L) nesta classe, a modelagem da relação entre CP e CQ se dá sem a utilização de métodos quantitativos, limitando-se a métodos qualitativos.

Como já dissemos, a classificação e codificação da característica foco do artigo será totalmente dependente da característica natureza do artigo (estrutura hierárquica), ou seja, teremos classes diferentes para os trabalhos quanti e qualitativos.

Para os trabalhos quantitativos, quanto ao foco do artigo, teremos as seguintes classes:

- Trabalhos nos quais não há modelo de otimização das variáveis de CP e CQ representaremos esta classe pelo número 1.

- Trabalhos nos quais ambas são otimizadas: variáveis de $\mathrm{CP}$ e CQ - representaremos esta classe pelo número 2 .

- Trabalhos nos quais são otimizadas somente variáveis de $\mathrm{CP}$ - representaremos esta classe pelo número 3 .

Já para os trabalhos qualitativos, teremos as seguintes classes, ainda de acordo com a característica foco do artigo:
- Relações entre qualidade e JIT/Kanban representaremos esta classe pelo número 1.

- Relações entre qualidade e ERP/MRP/ MRPII ou OPT - representaremos esta classe pelo número 2 .

- Relações entre variáveis de CP (capacidade, programação, tempo de entrega, nível de serviço, set up, redução de custos de produção, etc.) e variáveis de CQ (produto fora da especificação, gráfico de controle e inspeção) - representaremos esta classe pelo número 3 .

Quanto à característica objetivos do artigo, apresentaremos na Tabela 1 quais são eles e seus respectivos códigos, lembrando que tais objetivos foram obtidos em função das características do survey realizado.

De acordo com a classificação proposta, cada artigo recebe um código que identifica a classe a qual pertence. Um resumo do sistema de classificação e codificação proposto é mostrado nas Figuras 4 (quantitativos) e 5 (qualitativos).

Conforme salientado, o survey realizado sobre artigos que relacionam variáveis do $\mathrm{CP}$ a variáveis do CQ procurou ser o mais completo possível (45 artigos) e possibilitou criar o sistema de classificação e codificação de artigos. A fim de aplicarmos nosso sistema de classificação e codificação de artigos, realizamos a classificação e codificação dos artigos encontrados. Na Tabela 2 , mostramos que todos eles puderam ser classificados e codificados de acordo com o sistema proposto.

A codificação proposta usa / (barra) para a separação das características e - (traço) para a separação dos objetivos (quando houver mais de um). 
Tabela 1 - Códigos e respectivos objetivos de artigos que relacionam CP e CQ.

\begin{tabular}{|c|c|c|c|}
\hline Código & Objetivo & Código & Objetivo \\
\hline 01 & $\begin{array}{l}\text { Otimizar tamanho de lote de produção, o } \\
\text { qual inclui itens defeituosos. }\end{array}$ & 21 & $\begin{array}{l}\text { Otimizar a política de controle de qualidade (número e } \\
\text { periodicidade da amostra, número de aceitação para a } \\
\text { amostra). }\end{array}$ \\
\hline 02 & Otimizar tempo de setup. & 22 & Otimizar média de defeitos do processo. \\
\hline 03 & $\begin{array}{l}\text { Otimizar tamanho de lote de produção, o } \\
\text { qual não pode admitir itens defeituosos, } \\
\text { para diferentes políticas de inspeção. }\end{array}$ & 23 & $\begin{array}{l}\text { Mostrar que investimentos em redução de defeitos e } \\
\text { em redução de setup afetam a meta de qualidade da } \\
\text { empresa. }\end{array}$ \\
\hline 04 & Otimizar tempo de ciclo de produção. & 24 & $\begin{array}{l}\text { Mostrar que a integração entre automação, TQM e JIT } \\
\text { são essenciais. }\end{array}$ \\
\hline 05 & Otimizar tamanho de lote de produção. & 25 & $\begin{array}{l}\text { Mostrar a relação entre controle de qualidade e } \\
\text { sistemas MRP. }\end{array}$ \\
\hline 06 & Otimizar taxa de produção. & 26 & Mostrar relações entre tamanho de lote e qualidade. \\
\hline 07 & Otimizar tamanho de lote de retrabalho. & 27 & Mostrar pontos de interação entre CP e CQ. \\
\hline 08 & Otimizar custo de setup. & 28 & Calcular a qualidade média de saída de produtos. \\
\hline 09 & Otimizar fator de folga. & 29 & Otimizar custo de inspeção. \\
\hline 10 & $\begin{array}{l}\text { Otimizar investimento em redução de } \\
\text { setup. }\end{array}$ & 30 & Otimizar tamanho de lote de inspeção. \\
\hline 11 & $\begin{array}{l}\text { Otimizar probabilidade de o sistema sair } \\
\text { fora dos limites de controle. }\end{array}$ & 31 & $\begin{array}{l}\text { Otimizar quantidades de input para cada estágio do } \\
\text { processo em um sistema de produção seriado. }\end{array}$ \\
\hline 12 & $\begin{array}{l}\text { Otimizar investimento em melhoria de } \\
\text { qualidade. }\end{array}$ & 32 & Otimizar demanda durante períodos de falta. \\
\hline 13 & Otimizar política de produção. & 33 & Encontrar ótimo número de setups. \\
\hline 14 & Otimizar proporção de defeituosos. & 34 & $\begin{array}{l}\text { Mostrar a importância da qualidade (TQC) em um } \\
\text { programa JIT. }\end{array}$ \\
\hline 15 & Otimizar número de inspeções. & 35 & $\begin{array}{l}\text { Desenvolver relação entre um gráfico de controle de } \\
\text { qualidade para a média e a política de produção. }\end{array}$ \\
\hline 16 & Otimizar programação de inspeções. & 36 & $\begin{array}{l}\text { Encontrar um gráfico de controle para a média de } \\
\text { defeitos a fim de alcançar certo nível de estoque. }\end{array}$ \\
\hline 17 & Otimizar ponto de reabastecimento. & 37 & $\begin{array}{l}\text { Encontrar uma política de produção que maximiza o } \\
\text { nível de serviço, dado o gráfico de controle para a } \\
\text { média. }\end{array}$ \\
\hline 18 & Otimizar lead time de produção. & 38 & $\begin{array}{l}\text { Estudar os benefícios dos programas de melhoria } \\
\text { contínua na melhoria dos sistemas de CP e CQ. }\end{array}$ \\
\hline 19 & $\begin{array}{l}\text { Otimizar tamanho da amostra de } \\
\text { inspeção. }\end{array}$ & 39 & $\begin{array}{l}\text { Mostrar políticas de determinação do tamanho de lote } \\
\text { quando o rendimento na produção/compra é aleatória. }\end{array}$ \\
\hline 20 & $\begin{array}{l}\text { Otimizar número de aceitação para a } \\
\text { amostra de inspeção. }\end{array}$ & & \\
\hline
\end{tabular}




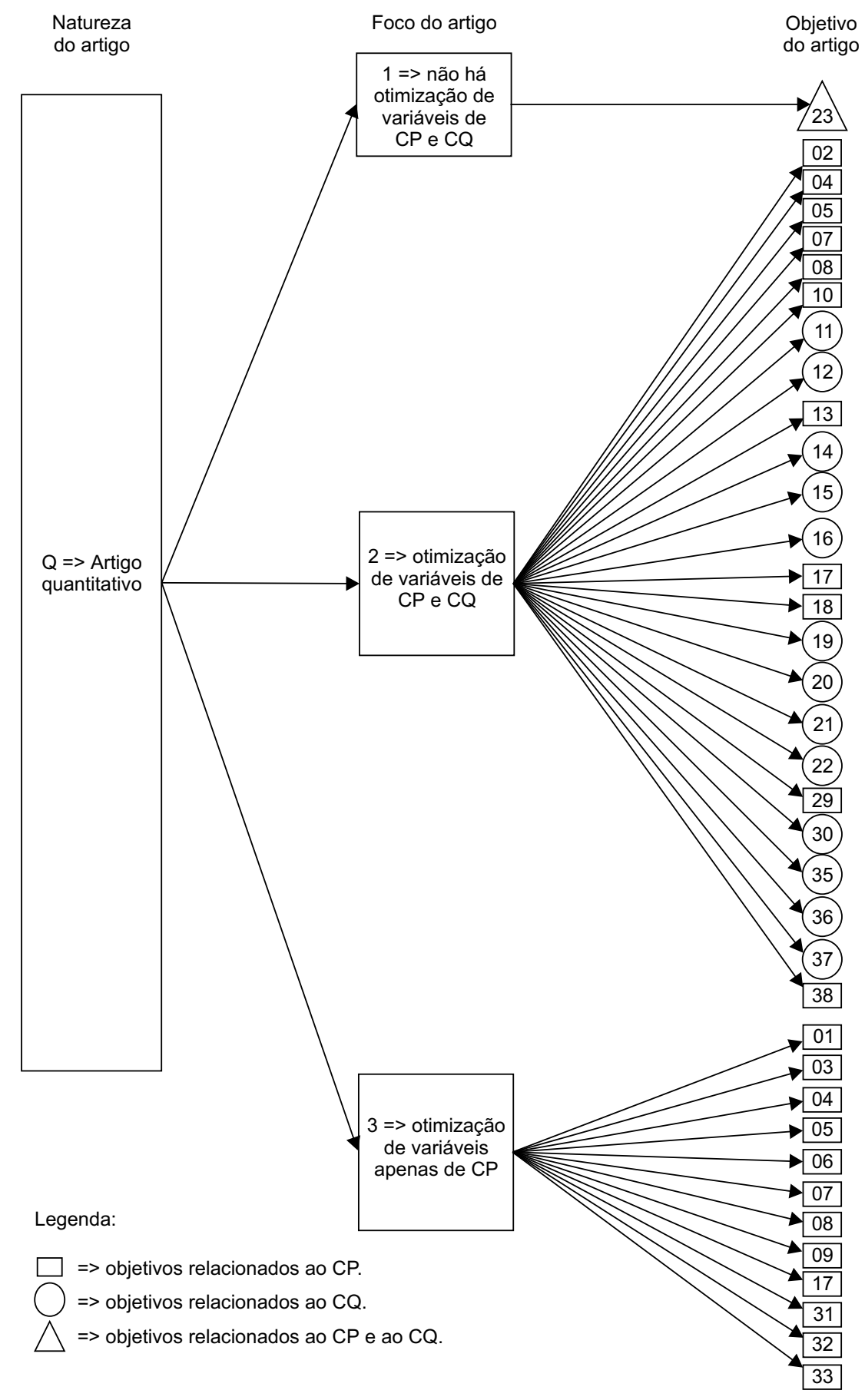

Figura 4 - Sistema de classificação e codificação de artigos relacionados ao CP e ao CQ para artigos de natureza quantitativa $(Q)$. 
Natureza

do artigo

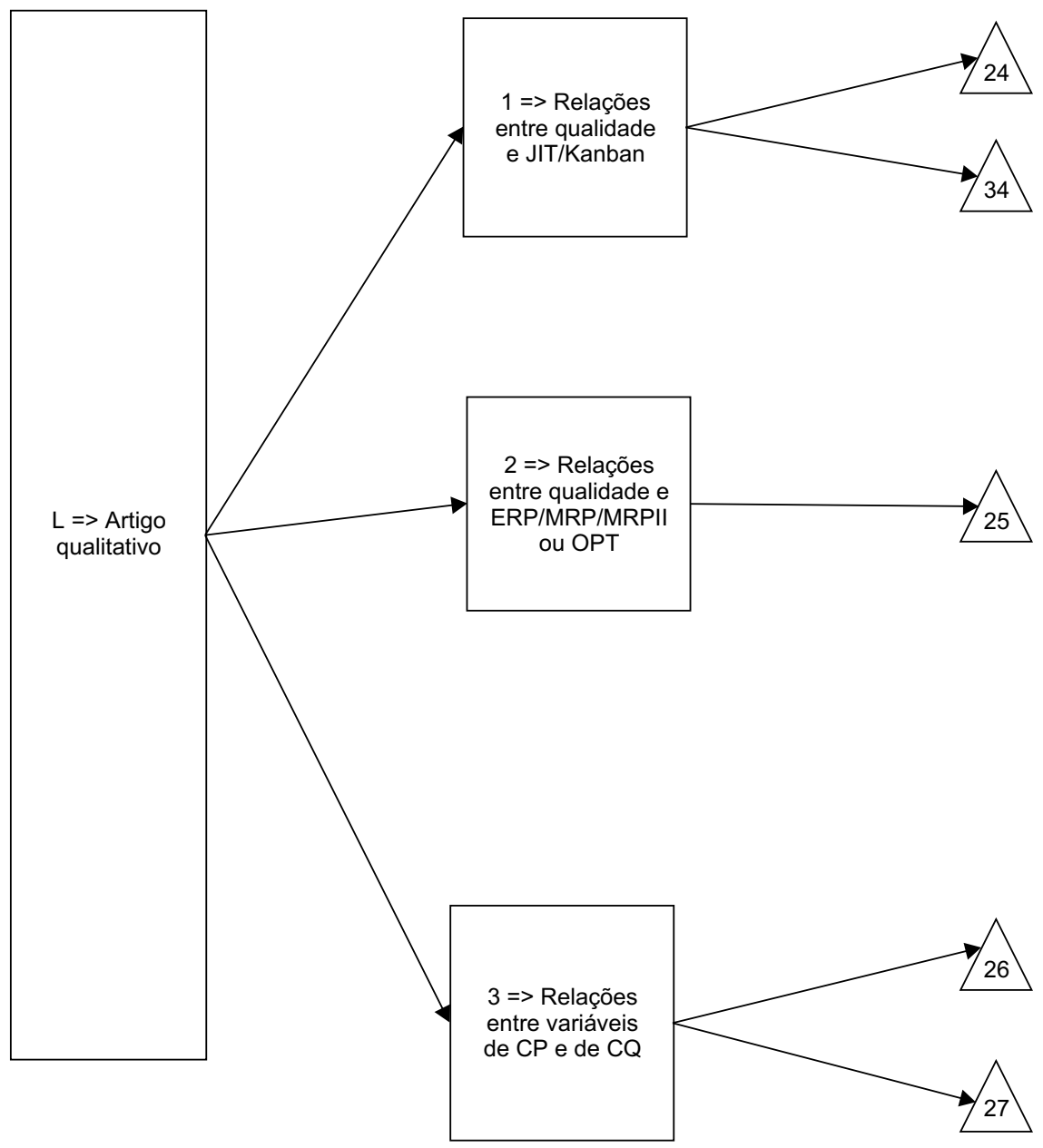

Legenda:

$\square=>$ objetivos relacionados ao $\mathrm{CP}$.
$=$ = objetivos relacionados ao $\mathrm{CQ}$.
$=>$ objetivos relacionados ao $\mathrm{CP}$ e ao $\mathrm{CQ}$.

Figura 5 - Sistema de classificação e codificação de artigos relacionados ao CP e ao CQ para artigos de natureza qualitativa (L). 
Tabela 2 - Codificação dos trabalhos que relacionam CP e CQ.

\begin{tabular}{|c|c|}
\hline Trabalho & Código \\
\hline Tapiero \& Hsu (1988) & Q (1988)/1/28 \\
\hline Yano \& Lee (1995) & Q (1995)/1/39 \\
\hline Wacker (1996) & Q (1996)/1/23 \\
\hline Fine (1986) & $\mathrm{Q}(1986) / 2 / 13-14$ \\
\hline Porteus (1986) & Q (1986)/2/05-08-11 \\
\hline Lee \& Rosenblatt (1987) & Q (1987)/2/04-15-16 \\
\hline Tapiero \& Hsu (1987) & $\mathrm{Q}(1987) / 2 / 05-29$ \\
\hline Keller \& Noori (1988) & $\mathrm{Q}(1988) / 2 / 05-08-11-17$ \\
\hline Peters et al. (1988) & $\mathrm{Q}(1988) / 2 / 05-17-19-20$ \\
\hline Goyal \& Gunasekaran (1989) & $\mathrm{Q}(1989) / 2 / 05-12$ \\
\hline Hsu \& Tapiero (1989) & Q (1989)/2/05-19 \\
\hline Hsu \& Tapiero (1990) & $\mathrm{Q}(1990) / 2 / 04-21$ \\
\hline Porteus (1990) & $\mathrm{Q}(1990) / 2 / 05-30$ \\
\hline Gupta \& Golhar (1991) & Q (1991)/2/05-07-22 \\
\hline Mehrez et al. (1991) & $\mathrm{Q}(1991) / 2 / 05-12$ \\
\hline Hwang et al. (1993) & $\mathrm{Q}(1993) / 2 / 02-04-08-14$ \\
\hline Moon (1994) & $\mathrm{Q}(1994) / 2 / 02-04-08-14$ \\
\hline Del Castillo (1995) & $\mathrm{Q}(1995) / 2 / 35-36-37-38$ \\
\hline Gunasekaran et al. (1995) & $\mathrm{Q}(1995) / 2 / 05-10-12$ \\
\hline Hong \& Hayya (1995) & $\mathrm{Q}(1995) / 2 / 10-12$ \\
\hline Chen \& Chung (1996) & Q (1996)/2/04-22 \\
\hline Lee et al. (1997) & $\mathrm{Q}(1997) / 2 / 05-14$ \\
\hline Cheung \& Leung (2000) & $\mathrm{Q}(2000) / 2 / 05-17-19-20$ \\
\hline Ouyang \& Chang (2000) & $\mathrm{Q}(2000) / 2 / 05-11-17-18$ \\
\hline Shih (1980) & Q (1980)/3/01 \\
\hline Arcelus et al. (1982) & $\mathrm{Q}(1982) / 3 / 05$ \\
\hline Gupta \& Chakraborty (1984) & $\mathrm{Q}(1984) / 3 / 05-07$ \\
\hline Lee \& Rosenblatt (1985) & $\mathrm{Q}(1985) / 3 / 03$ \\
\hline Mak (1985) & Q (1985)/3/04-05-32 \\
\hline Rosenblatt \& Lee (1986a) & Q (1986)/3/04-08 \\
\hline Rosenblatt \& Lee (1986b) & Q (1986)/3/04-33 \\
\hline Moinzadeh \& Lee (1987) & Q (1987)/3/05-17 \\
\hline Lee \& Yano (1988) & Q (1988)/3/31 \\
\hline Chand (1989) & $\mathrm{Q}(1989) / 3 / 05-33$ \\
\hline Groenevelt et al. (1992) & $\mathrm{Q}(1992) / 3 / 05$ \\
\hline Khouja \& Mehrez (1994) & Q (1994)/3/06-07 \\
\hline Murthy \& Ma (1996) & Q (1996)/3/09 \\
\hline Urban (1998) & Q (1998)/3/05 \\
\hline Hohner (1988) & $\mathrm{L}(1988) / 1 / 34$ \\
\hline Kagemann (1990) & $\mathrm{L}(1990) / 1 / 24$ \\
\hline Murthy \& Ma (1991) & $\mathrm{L}(1991) / 2 / 25$ \\
\hline Inman (1994) & $\mathrm{L}(1994) / 3 / 26$ \\
\hline Jokinen et al. (1995) & $\mathrm{L}(1995) / 3 / 27$ \\
\hline Arentsen et al. (1996) & $\mathrm{L}(1996) / 3 / 27$ \\
\hline Van Der Bij \& Van Ekert (1999) & $\mathrm{L}(1999) / 3 / 27$ \\
\hline
\end{tabular}




\section{Revisão bibliográfica}

Nesta seção estruturamos o survey realizado dentro das relações entre $\mathrm{CP}$ e $\mathrm{CQ}$, com base em nosso sistema de classificação e codificação de artigos (note que a numeração desta seção segue a lógica de nosso sistema de classificação e codificação de artigos). Esse survey procurou ser o mais completo possível. Porém é importante deixar claro que seu foco foi exclusivamente os trabalhos que relacionam explicitamente $\mathrm{CP}$ e $\mathrm{CQ}$, excluindo todos os artigos que tratam de rendimentos aleatórios na produção/compra sem especificar se estas são causadas por problemas ligados ao CQ. Uma revisão do problema de determinação do tamanho de lote com rendimentos aleatórios na produção/compra sem a preocupação se estes são ou não relacionados à qualidade é encontrada em Yano \& Lee (1995). Esse artigo entrou em nosso survey por abordar alguns artigos que se referem à qualidade, porém, ele trata de rendimentos aleatórios sob um aspecto mais amplo que esta revisão, não se focando nos aspectos da qualidade ou na relação entre CP e CQ.

\subsection{Artigos quantitativos (Q/........)}

Nesta seção apresentamos os 45 artigos quantitativos encontrados em nosso survey, lembrando que estes podem ser subdivididos, de acordo com o foco do artigo, em: trabalhos nos quais não há modelo de otimização das variáveis de $\mathrm{CP}$, trabalhos nos quais são otimizadas variáveis tanto de CP quanto de CQ e trabalhos nos quais são otimizadas somente variáveis de $\mathrm{CP}$ - representaremos essa classe pelo número 3 .

\subsubsection{Artigos quantitativos nos quais não há otimização de variáveis de CP e CQ (codificação $=>$ Q/1/........)}

Os artigos de Tapiero \& Hsu (1988), Yano \& Lee (1995) e Wacker (1996) são de natureza quantitativa, porém não se preocupam em buscar um modelo otimizante para variáveis de $\mathrm{CP}$ e CQ.

O artigo de Tapiero \& Hsu (1988) fornece um conjunto de equações para calcular a qualidade média de saída das peças em um sistema flexível de manufatura. Já Wacker (1996) mostra que o tamanho de lote de produção (afetado pelo tempo de setup), juntamente com programas de redução de defeitos e programas de manutenção preventiva, afetam a taxa de defeitos que, por sua vez, afeta o lead time da manufatura por meio de um efeito direto no tempo de retrabalho; portanto, investimentos no controle dessas variáveis (taxa de defeitos e tempo de setup) afetam a meta de qualidade de uma empresa de manufatura.

\subsubsection{Artigos quantitativos nos quais são otimizadas variáveis de CP e CQ (codificação $=>Q / 2 / . . . . . .$.}

Dentro desta classe encontramos 21 artigos de nosso survey. É interessante notar que a minimização dos custos totais parece ser o ponto em comum para a grande maioria desses artigos. Essa característica aparece em 17 dos 21 artigos. Apesar disso, podemos notar dois aspectos diferenciais entre eles: a composição dos custos totais e as variáveis otimizadas. Por exemplo, enquanto Lee et al. (1997) consideram nos custos totais os custos de armazenagem, de processamento, de perda de lucro, da perda de Tagushi e de setup, encontrando um ótimo para o tamanho de lote (variável de $\mathrm{CP}$ ) e para a proporção de defeituosos (variável de CQ); Hsu \& Tapiero (1989) consideram os custos totais formados pelos custos de inspeção, de processamento, de refugo, de armazenagem e de espera. Esse autor também otimiza a variável tamanho de lote, porém a variável de CQ otimizada é o tamanho da amostra de inspeção.

Ainda dentro dessa classe de artigos, que se utiliza da minimização dos custos totais na obtenção do valor ótimo para certas variáveis, 
temos os artigos de Porteus (1986) e Keller \& Noori (1988), os quais otimizam o tamanho do lote de produção, o custo de setup (variável de $\mathrm{CP}$ ) e a probabilidade de o sistema extrapolar os limites de controle (variável de CQ). Podemos dizer que Porteus (1986) foi o primeiro autor a tratar variáveis de CQ como variáveis de decisão, ou seja, passou a se preocupar com a otimização também de variáveis de CQ e não somente de CP. Keller \& Noori (1988) ampliam um pouco o modelo de Porteus (1986), encontrando também um valor ótimo para o ponto de reabastecimento de estoque (variável de $\mathrm{CP}$ ). O artigo de Ouyang \& Chang (2000) apresenta modelagem semelhante ao modelo de Keller \& Noori (1988), porém as variáveis otimizadas são, além do tamanho de lote de produção, da probabilidade de o sistema extrapolar os limites de controle e do ponto de reabastecimento do estoque, o lead time de produção (variável de $\mathrm{CP}$ ), o qual é considerado estocástico e nãodeterminístico, como no modelo de Keller \& Noori (1988).

Outros artigos que tratam de objetivos semelhantes são: Goyal \& Gunasekaran (1989) e Mehrez et al. (1991), os quais tratam da otimização do tamanho de lote de produção e do investimento em melhoria de qualidade (variável de CQ); Hwang et al. (1993) e Moon (1994), os quais tratam da otimização do tempo de setup (variável de $\mathrm{CP}$ ), do tempo de ciclo de produção (variável de CP), do custo de setup e da proporção de defeituosos [o modelo de Moon (1994) é complementar ao de Hwang et al. (1993), adicionando a este algumas restrições]; e Peters et al. (1988) e Cheung \& Leung (2000), os quais otimizam as variáveis tamanho de lote de produção, ponto de reabastecimento do estoque, tamanho da amostra de inspeção e número de aceitação para a amostra de inspeção (variável de CQ). Também os trabalhos de Gunasekaran et al. (1995) e Hong \& Hayya (1995) tratam de objetivos comuns: otimização do investimento em redução do setup (variável de $\mathrm{CP}$ ) e do investimento em melhoria de qualidade (variável de CQ). A particularidade entre esses dois modelos é que o modelo de Gunasekaran et al.
(1995) também otimiza o tamanho de lote de produção. Finalizando os artigos que utilizam modelos de minimização dos custos totais, temos Lee \& Rosenblatt (1987), que priorizam a otimização do tempo de ciclo de produção, do número e da programação das inspeções (variáveis de CQ); Tapiero \& Hsu (1987), que otimizam o tamanho de lote de produção e o custo de inspeção (variável de CQ); Porteus (1990), o qual busca o ótimo lote de produção e o ótimo lote de inspeção para uma gama variada de atrasos na inspeção; e Gupta \& Golhar (1991), os quais otimizam, além do tamanho de lote de produção, o tamanho de lote de retrabalho (variável de CQ) e a média de defeitos no processo (variável de CQ).

Os outros quatro artigos quantitativos que realizam otimização tanto de variáveis de $\mathrm{CP}$ quanto de CQ não utilizam modelos de minimização de custos totais para realizar a otimização das variáveis. Três deles, Fine (1986), Hsu \& Tapiero (1990) e Chen \& Chung (1996), optam por maximizar os lucros. Fine (1986) confronta a teoria da otimização da proporção de defeituosos com a teoria do zero defeito. Um modelo que soma os efeitos da aprendizagem ao modelo de otimização da proporção de defeitos é desenvolvido a fim de tentar reconciliar as duas teorias. Nesse modelo, a variável de CP tamanho de lote é otimizada em conjunto com a variável de CQ proporção de defeituosos. Hsu $\&$ Tapiero (1990) encontram ótimos tempos de ciclo de produção e política de controle de qualidade (número e periodicidade da amostra, número de aceitação para a amostra). Já Chen \& Chung (1996) encontram, além do ótimo tempo de ciclo, a ótima média de defeitos no processo. Outro artigo que não recorre à minimização dos custos é o trabalho de Del Castillo (1995), o qual não se preocupa em realizar modelagens de custo. Esse autor desenvolve relação analítica entre as variáveis de projeto de um gráfico de controle para a média e variáveis da produção para um sistema de produção de um item único, de demanda estocástica. É encontrado um projeto de gráfico de média para alcançar certo nível de serviço para 
uma política de produção dada ou uma política de produção que maximiza o nível de serviço dado um projeto de gráfico de controle para a média.

\subsubsection{Artigos quantitativos nos quais são otimizadas apenas variáveis de $\mathrm{CP}$ ( codificação $=>Q / 3 /$........)}

Dentro desta categoria, nosso survey apresentou 14 artigos. Todos eles utilizam métodos de minimização de custos totais para a otimização de amplo número de variáveis de $\mathrm{CP}$. Como nos artigos que mostramos na seção anterior, o objetivo que mais aparece nessa classe é a otimização do tamanho de lote. Os artigos de Shih (1980), Arcelus et al. (1982), Gupta \& Chakraborty (1984), Rosenblatt \& Lee (1985), Mak (1985), Moinzadeh \& Lee (1987), Chand (1989), Groenevelt et al. (1992) e Urban (1998) tratam da otimização do tamanho de lote de produção/compra. Destes, alguns apresentam modelagens especiais para o tamanho de lote ótimo, por exemplo, Shih (1980), o primeiro autor que levou em conta variáveis de CQ em modelos otimizantes de $\mathrm{CP}$, se preocupa exclusivamente com a otimização de um tamanho de lote, o qual pode incluir itens defeituosos; já Rosenblatt \& Lee (1985) não admitem itens defeituosos em seu lote otimizado. Além da otimização do tamanho de lote, a maioria desses artigos trata também de outros objetivos: Gupta \& Chakraborty (1984) encontram um tamanho otimizado para o lote de retrabalho. Mak (1985) desenvolve um modelo matemático para um sistema de estoque no qual o número de unidades de qualidade aceitável em um lote de ressuprimento é incerto, encontrando o ótimo tempo de ciclo de produção e as quantidades ótimas de input para cada estágio do processo de tal sistema. Moinzadeh \& Lee (1987) também desenvolvem um modelo para um sistema de estoque cujos itens de ressuprimento não são todos de qualidade perfeita; eles otimizam o ponto de reabastecimento para tal sistema. Já Chand (1989) reconhece os benefícios de tamanhos de lote pequenos pela redução de custos de setup, em razão da aprendizagem do trabalhador e da melhoria na qualidade do processo. Esse autor encontra uma freqüência ótima de setups.

Os outros artigos dessa classe não tratam diretamente da otimização do tamanho do lote de produção/compra. Rosenblatt \& Lee (1986a) encontram em seu modelo um ótimo tempo de ciclo de produção e um ótimo custo de setup. Também Rosenblatt \& Lee (1986b) se preocupam em otimizar o tempo de ciclo de produção, porém, em vez da otimização do custo de setup é a freqüência de setups que é otimizada. Khouja \& Mehrez (1994) apresentam um modelo no qual o tempo transcorrido até a mudança de estado do processo é dependente da taxa de produção, ou seja, quanto maior a taxa de produção, mais rapidamente um processo tende a extrapolar os limites de controle. Nesse trabalho as variáveis otimizadas são o tamanho do lote de retrabalho e o custo de setup. Murthy \& Ma (1996) entendem que, em decorrência de variações de qualidade, há fração de itens defeituosos em um lote. Essa fração de defeituosos faz com que a quantidade de output em cada nível e estágio de produção tenha de ser planejada (no MRP) com um fator de folga (CP), a fim de que a demanda seja atingida. Esses autores tentam em seu trabalho otimizar o fator de folga.

\subsection{Artigos qualitativos (L/........)}

Nesta seção apresentamos os sete artigos qualitativos encontrados em nosso survey. Eles se dividem em três características conforme o foco do artigo: artigos que relacionam a qualidade e o sistema JIT/Kanban; artigos que relacionam a qualidade e os sistemas ERP/MRP/ MRPII ou OPT e, finalmente, aqueles que apresentam relações entre variáveis de CP (capacidade, programação, tempo de entrega, nível de serviço, setup, redução de custos de produção, etc.) e variáveis de CQ (produto fora da especificação, gráfico de controle e inspeção). 


\subsubsection{Artigos qualitativos que relacionam a qualidade e os sistemas JIT/ Kanban $($ codificação $=>\mathrm{L} / \mathbf{1} /$.........)}

Nesta categoria temos os artigos de Hohner (1988), que apresenta a metodologia do controle da qualidade total (TQC), a qual o autor defende ser a base de uma implementação JIT de sucesso, mostrando que o TQC é um passo crucial dentro dos programas JIT; e o trabalho de Kagemann (1990), para o qual a integração entre automação, gerenciamento total da qualidade (TQM) e JIT são essenciais para obter os objetivos de qualidade e produtividade necessários atualmente.

\subsubsection{Artigos qualitativos que relacionam a qualidade e os sistemas ERP/ MRP/MRPII ou OPT (codificação $=>\mathrm{L} / 2 / . . . . .$. .)}

Nesta categoria há grande diversidade de trabalhos, principalmente ligando os sistemas MRP/MRPII e as incertezas de qualidade. Em nosso survey, classificamos somente o trabalho de Murthy \& Ma (1991), o qual constitui uma completa revisão bibliográfica de trabalhos que exploram o sistema MRP com incertezas devidas à qualidade. Esses autores também mostram como estão tentando minimizar ou corrigir os problemas da qualidade que afetam o bom funcionamento dos sistemas MRP.

\subsubsection{Artigos qualitativos que relacionam variáveis de $C P$ com variáveis de CQ $($ codificação $=>\mathrm{L} / 3 /$........)}

Nesta categoria foram encontrados quatro trabalhos. Inman (1994) propõe a redução do lote de produção para melhoria da qualidade, descrevendo as relações entre redução do tamanho do lote e melhoria da qualidade. Os outros três trabalhos mostram pontos de interação entre CP e CQ. Jokinem et al. (1995) trata da integração do controle da qualidade e operações de processo em um laboratório, utilizando modernas soluções computadorizadas. Um modelo geral para a integração do gerenciamento da produção e da qualidade é desenvolvido. Arentsen et al. (1996) tratam da integração do controle da qualidade e do controle de chão de fábrica. Com base na série de normas ISO, cinco tarefas da qualidade são identificadas, as quais são relacionadas ao controle de chão de fábrica. Atenção especial é dada às atividades de inspeção, que, para esses autores, é a parte mais importante do CQ. Também é discutido nesse trabalho a implementação de um módulo de controle de qualidade. Finalmente, Van Der Bij \& Van Ekert (1999) descrevem um estudo qualitativo da interação entre sistemas de controle da produção e controle da qualidade com base em quatro estudos de caso. Esses autores defendem que a boa performance de um geralmente influencia ou frustra a performance do outro. Os autores se referem a controle de produção e controle da qualidade em separado, mas ambos influenciando o sistema de controle em uma organização.

\section{Análise dos trabalhos por meio do sistema proposto}

Será realizada nesta seção a análise dos trabalhos que relacionam CP e CQ à luz de nosso sistema de classificação e codificação.

A primeira constatação a respeito dos trabalhos é que há um número bem maior de trabalhos quantitativos $(84,45 \%)$ que relacionam CP e CQ do que qualitativos $(15,55 \%)$.

Vemos também que a grande maioria dos trabalhos quantitativos se refere à otimização de variáveis (nesse caso, a característica foco do artigo para trabalhos quantitativos assume valores 2 ou 3), e somente três artigos não apresentam modelo de otimização de variáveis. Portanto, entre os artigos quantitativos, 7,89\% (3) não utilizam qualquer modelo otimizante, $36,84 \%$ (14) otimizam somente variáveis de $\mathrm{CP}$ e a maioria, ou seja, $55,26 \%$ (21), otimiza tanto variáveis de $\mathrm{CP}$ quanto de CQ. 
No universo de trabalhos quantitativos, podemos perceber que entre os trabalhos que utilizam algum modelo de otimização de variáveis, parece haver maior tendência a objetivos relacionados ao $\mathrm{CP}$ e não ao CQ (100\% dos trabalhos apresentam objetivos relacionados à otimização de, ao menos, alguma variável de $\mathrm{CP}$, enquanto somente $60 \%$ dos trabalhos otimizam variáveis de CQ).

Os artigos qualitativos são mais abrangentes no sentido de tratarem de objetivos tanto de $\mathrm{CP}$ quanto de CQ (veja Figura 5), enquanto apenas um artigo quantitativo trata de objetivos de $\mathrm{CP}$ e CQ (veja triângulo na Figura 4 e na Tabela 3), mas não otimiza variáveis. Quando há otimização de variáveis os objetivos são relacionados ao $\mathrm{CP}$ ou ao CQ.

Ainda em relação à característica objetivo do artigo, construímos a Tabela 3, a qual relaciona, tanto para os trabalhos quantitativos quanto para os qualitativos, o objetivo do artigo com a freqüência em que este objetivo aparece nos artigos.

Tabela 3 - Relação entre os objetivos dos artigos e a freqüência em que estes objetivos aparecem nos artigos.

\begin{tabular}{cccccc}
\hline Objetivo & $\begin{array}{c}\text { Frequiência em que } \\
\text { o objetivo aparece }\end{array}$ & $\begin{array}{c}\text { Freqüência } \\
\text { relativa }\end{array}$ & Objetivo & $\begin{array}{c}\text { Frequiência em que } \\
\text { o objetivo aparece }\end{array}$ & $\begin{array}{c}\text { Freqüência } \\
\text { relativa }\end{array}$ \\
\hline 1 & 1 & $2,22 \%$ & 21 & 1 & $2,22 \%$ \\
2 & 2 & $4,44 \%$ & 22 & 2 & $4,44 \%$ \\
3 & 1 & $2,22 \%$ & 23 & 1 & $2,22 \%$ \\
4 & 8 & $17,76 \%$ & 24 & 1 & $2,22 \%$ \\
5 & 19 & $42,19 \%$ & 25 & 1 & $2,22 \%$ \\
6 & 1 & $2,22 \%$ & 26 & 1 & $2,22 \%$ \\
7 & 3 & $6,66 \%$ & 27 & 3 & $6,66 \%$ \\
8 & 5 & $11,06 \%$ & 28 & 1 & $2,22 \%$ \\
9 & 1 & $2,22 \%$ & 29 & 1 & $2,22 \%$ \\
10 & 2 & $4,44 \%$ & 30 & 1 & $2,22 \%$ \\
11 & 3 & $6,66 \%$ & 31 & 1 & $2,22 \%$ \\
12 & 4 & $8,88 \%$ & 32 & 1 & $2,22 \%$ \\
13 & 1 & $2,22 \%$ & 33 & 2 & $4,44 \%$ \\
14 & 4 & $8,88 \%$ & 34 & 1 & $2,22 \%$ \\
15 & 1 & $2,22 \%$ & 35 & 1 & $2,22 \%$ \\
16 & 1 & $2,22 \%$ & 36 & 1 & $2,22 \%$ \\
17 & 5 & $11,06 \%$ & 37 & 1 & $2,22 \%$ \\
18 & 1 & $2,22 \%$ & 38 & 1 & $2,22 \%$ \\
19 & 3 & $6,66 \%$ & 39 & 1 & $2,22 \%$ \\
20 & 2 & $4,44 \%$ & & & \\
\hline
\end{tabular}


Vemos na Tabela 3 que o objetivo 5, ou seja, a otimização do lote de produção, é tratado em 42,19\% dos artigos, seguido pelo objetivo 4, o qual é a otimização do tempo de ciclo de produção (tratado em 17,76\% dos artigos). Ambos os objetivos, na verdade, estão intimamente ligados, pois a otimização de um leva à otimização do outro (são diretamente proporcionais), lembrando que nesses artigos tempo de ciclo é definido como tempo total de produção de um lote de peças. Outros objetivos bastante citados nos artigos que relacionam $\mathrm{CP}$ e CQ, como mostra a Tabela 3, são: otimização dos custos de setup e do ponto de reabastecimento (ambos aparecem em $11,06 \%$ dos trabalhos) e otimização do investimento em melhoria de qualidade e da proporção de defeituosos (ambos aparecem em $8,88 \%$ dos trabalhos).

\section{Conclusões}

Podemos dizer que a proposição e a aplicação de nosso sistema de classificação e codificação de artigos (SCCA) se prestou muito bem a seus objetivos iniciais, pois foi extremamente útil na estruturação da revisão bibliográfica sobre o tema (mostrado na Seção 4) e possibilitou uma análise geral das relações entre CP e CQ (mostrado na Seção 5). Esta análise, por sua vez, propicia maior esclarecimento e comunicação das pesquisas atuais que relacionam $\mathrm{CP}$ e $\mathrm{CQ}$ e, ao mesmo tempo, serve de base para sugestão de novas áreas de estudo e pesquisa dentro do campo das relações entre CP e CQ. Essas áreas são exatamente aquelas que, por ocasião da análise dos trabalhos, identificamos ser as menos exploradas até o momento, sendo assuntos interessantes do ponto de vista acadêmico.

Primeiramente, podemos dizer que há na literatura grande número de trabalhos que relacionam quantitativamente $\mathrm{CP}$ e $\mathrm{CQ}$, porém, há poucos trabalhos qualitativos sobre as relações entre CP e CQ. Portanto, sem dúvida, essa é uma área a ser explorada. $\mathrm{O}$ emprego do estudo de caso ou da pesquisa de campo nas relações entre
$\mathrm{CP}$ e CQ pode demonstrar muitas relações ainda ocultas entre essas duas instituições.

Dentre os trabalhos quantitativos, fizemos uma constatação bastante interessante. Na década de 80, havia maior concentração de trabalhos que buscavam somente a otimização de variáveis de CP, entendendo as variáveis de CQ apenas como variáveis que influenciavam o modelo, não como variáveis de decisão. Já na década de 90 , apesar desse tipo de trabalho continuar a existir, de modo geral houve grande crescimento de trabalhos que realizam ambas as otimizações, tanto das variáveis de $\mathrm{CP}$ quanto de $\mathrm{CQ}$, ou seja, as variáveis de CQ começaram a se tornar também variáveis de decisão. Portanto, isso nos leva a crer que a tendência atual no campo de pesquisa das relações quantitativas entre $\mathrm{CP}$ e $\mathrm{CQ}$ é a otimização tanto de variáveis de $\mathrm{CP}$ quanto de CQ. Prova disso são os recentes trabalhos de Ouyang \& Chang (2000) e Cheung \& Leung (2000).

Outra conclusão importante que resulta da análise dos trabalhos que relacionam $\mathrm{CP}$ e $\mathrm{CQ}$, à luz de nosso sistema de classificação e codificação, é em relação aos objetivos dos trabalhos. Quando há otimização de variáveis, os objetivos são relacionados ao CP ou ao CQ; há, naturalmente, grande espaço para trabalhos que otimizem variáveis e contemplem tanto objetivos de CP quanto de CQ. Notamos que, dentre os trabalhos quantitativos, assuntos como otimização do tamanho de lote e tempo de ciclo de produção já foram tratados em muitos artigos (ver Tabela 3). Outros objetivos, como otimização dos custos de setup, do ponto de reabastecimento e da proporção de defeituosos (taxa de refugo), também aparecem em considerável número de artigos. Porém, há grande número de objetivos tratados em pouquíssimos artigos, como, por exemplo, a otimização do tempo de setup ou a otimização da programação das inspeções. Além disso, quaisquer outros objetivos relevantes relacionados a CP e CQ que não aparecem na Tabela 3 também são áreas interessantes de pesquisa. Porém, isso não significa que 
os objetivos mais pesquisados, como os citados anteriormente, não são interessantes, pois, apesar de serem em maior número, em relação aos trabalhos que envolvem relação entre $\mathrm{CP}$ e CQ, ainda nos parecem ser, em termos absolutos, bastante reduzidos se comparados a outros campos de pesquisa da engenharia de produção.

Finalizando este artigo, podemos indicar outras duas possíveis utilidades do sistema proposto. A primeira delas é auxiliar na verificação de plágio entre artigos. Para haver plágio, o código deve ser o mesmo, porém se o código for o mesmo não necessariamente haverá plágio. Por exemplo, Hwang et al. (1993) e Moon (1994) apresentam o mesmo código, mas não há plágio, já que em Moon (1994) acrescentam-se algumas restrições inexistentes em Hwang et al. (1993). Por fim, sugerimos que a idéia da construção do SCCA pode ser expandida para outras áreas do conhecimento (a metodologia mostrada na Figura 2 pode ser usada em outras áreas da ciência). Estamos trabalhando para estruturar uma metodologia geral de classificação e codificação de artigos e análise de um tema de pesquisa genérico, com base na metodologia aqui apresentada.

\section{Agradecimentos}

Agradecemos à Fapesp pelo apoio financeiro e aos três referees anônimos que contribuíram com valiosas sugestões.

\section{Referências Bibliográficas}

ARCELUS, F. J.; BANERJEE, P. K.; CHANDRA, R. Optimal production run for a normally distributed quality characteristic exhibiting nonnegative shifts in process mean and variance. IIE Transactions, v. 14, n. 2, p. 90-98, 1982.

ARENTSEN, A. L.; TIEMERSMA, J. J.; KARS, H. $\mathrm{J}$. J. The integration of quality control and shop floor control. International Journal Computer Integrated Manufacturing, v. 9, n. 2, p. 113-130, 1996.

BURBIDGE, J. L. Production control: a universal conceptual framework. Production Planning \& Control, v. 1, p. 3-16, 1990.

CHAND, S. Lot sizes and setup frequency with learning in setups and process quality. European Journal of Operational Research, v. 42, p. 190-202, 1989.

CHEN, S. L.; CHUNG, K. J. Determination of the optimal production run and most profitable process mean for a production process. International Journal of Production Research, v. 34, n. 7, p. 2051-2058, 1996.

CHEUNG, K. L.; LEUNG, K. F. Coordinating replenishments in a supply chain with quality control considerations. Production Planning \& Control, v. 11, n. 7, p. 697-705, 2000.

DEL CASTILLO, E. Relations between X control chart design variables and production control. International Journal of Production Research, v. 33, n. 10, p. 2709-2721, 1995.
FERNANDES, F. C. F. Concepção de um sistema de controle de produção para a manufatura celular. 1991. Tese (Doutorado) - Escola de Engenharia de São Carlos, Universidade de São Paulo, São Carlos.

FERNANDES, F. C. F.; MACCARTHY, B. L. Production planning and control: the gap between theory and practice in the light of modern manufacturing concepts. In: INTERNATIONAL CONFERENCE ON CAD/CAM, ROBOTICS \& FACTORIES OF THE FUTURE (CARS\& FOD'99), 15., 1999, Aguas de Lindóia. Proceedings... Águas de Lindóia, 1999.

FINE, C. H. Quality improvement and learning in productive systems. Management Science, v. 32, p. 1301-1315, 1986.

GOOD, I. J. Categorization of classification. In: Mathematics and computer science in medicine and biology. London: HMSO, p. 115-128, 1965.

GOYAL, S. K.; GUNASEKARAN, A. Effect of dynamic process quality control on the economics of production. Process Quality Control, p. 69-77, 1989.

GROENEVELT, H.; PINTELON, L.; SEIDMANN, A. Production lot sizing with machine breakdowns. Management Science, v. 38, n. 1, p. 104-123, 1992.

GROOVER, M. P.; ZIMMERS JR., E. W. Group technology. CAD/CAM: computer aided design and manufacturing. New Jersey: Prentice Hall, 1984. 
GUNASEKARAN, A.; KORUKONDA, A. R.; VIRTANEN, I.; YLI-OLLI, P. Optimal investment and lot-sizing policies for improved productivity and quality. International Journal of Production Research, v. 33, n. 1, p. 261-278, 1995.

GUPTA, T.; CHAKRABORTY, S. Looping in multistage production system. International Journal of Production Research, v. 22, p. 299-311, 1984.

GUPTA, T.; GOLHAR, D. Y. Determination of optimal lot sizing parameters and a controllable process mean for a production system. International Journal of Production Research, v. 29, p. 821-834, 1991.

HICKS, P. E. Industrial engineering and management a new perspective. McGraw Hill, 1994.

HOHNER, G. JIT/TQC: integrating product design with shop floor effectiveness. Industrial Engineering, v. 20, n. 9, p. 42-28, 1988.

HONG, J. D.; HAYYA, J. C. Joint investment in quality improvement and setup reduction. Computers \& Operations Research, v. 22, p. 567-574, 1995.

HSU, L. F.; TAPIERO, C. S. Quality control of the M/G/1 queue. European Journal of Operational Research, v. 42, p. 88-100, 1989.

HSU, L. F.; TAPIERO, C. S. Economic model for determining the optimal quality and process control policy in a queue - like Production system. International Journal of Production Research, v. 28, n. 8, p. 1447-1457, 1990.

HWANG, H.; KIM, D.; KIM, Y. Multi-product economic lot size models with investment costs for setup reduction and quality improvement. International Journal of Production Research, v. 31, p. 691-703, 1993.

ISHIKAWA, K. What is quality control? The japanese way. Tokio, Japan: Prentice Hall of Japan, Inc., 1981.

INMAN, R. A. The impact of lot size reduction on quality. Production and Inventory Management Journal, First Quarter, 1994.

JOKINEN, P.; ASLJOKI, K.; DRESSLER, N. Integration on quality control and process operations - a case. Process Control and Quality, v. 6, p. 209-218, 1995.

KAGEMANN, L. E. Integrating automated PCB component assembly with quality planning. Electronic Manufacturing, v. 36, n. 1, p. 22-25, jan. 1990.

KELLER, G.; NOORI, H. Impact of investing in quality improvement on the size model. OMEGA International Journal of Management Sciences, v. 15 , p. 595-601, 1988.
KHOUJA, M.; MEHREZ, A. Economic production lot size model with variable production rate and imperfect quality. Journal of the Operational Research Society, v. 45, n. 12, p. 1405-1417, 1994.

LEE, H. L.; CHANDRA, M. J.; DELEVEAUX, V. J. Optimal batch size and investment in multistage production systems with scrap. Production Planning \& Control, v. 8, n. 6, p. 586-596, 1997.

LEE, H. L.; ROSENBLATT, M. J. Optimal inspection and ordering policies for products with imperfect quality. IIE Trans., v. 17, n. 3, p. 284-289, 1985.

LEE, H. L.; ROSENBLATT, M. J. Simultaneous determination of production cycles and inspection schedules in a production system. Management Science, v. 33, n. 9, p. 1125-1135, 1987.

LEE, H. L.; YANO, C. A. Production control in multistage systems with variable yield losses. Operations Research, v. 36, n. 2, p. 269-278, 1988.

MAK, K. L. Inventory control of defective products when the demand is partially captive. International Journal Production Research, v. 23, n. 3, p. 533542, 1985.

MEHREZ, A.; MYERS, B. L.; KHOUJA, M. J. Quality and Inventory issues within the newsboy problem. Computers and Operations Research, v. 8, n. 5, 1991.

MOINZADEH, K.; LEE, H. L. A continuous-review inventory model with constant resupply time and defective items. Naval Research Logistics, v. 34, p. 457-467, 1987.

MOON, I. Multi-product economic lot size models with investments costs for setup reduction and quality improvement: review and extensions. International Journal of Production Research, v. 32, p. 2795-2801, 1994.

MURTHY, D. N. P.; MA, L. MRP with uncertainty a review and some extension. International Journal Production Economics, v. 25, n. 51, 1991.

MURTHY, D. N. P.; MA, L. Material planning with uncertain product quality. Production Planning \& Control, v. 7, n. 6, p. 566-576, 1996.

OPITZ, H.; EVERSHEIN, W.; WIENDAHL, H. P. Workpiece classification and its industrial application. Int. J. Mach. Tool Des. Res., v. 9, Pergamon Press, 1969.

OUYANG, L. Y.; CHANG, H. C. Impact of investing in quality improvement on $(\mathrm{Q}, \mathrm{r}, \mathrm{L})$ model involving the imperfect production process. Production Planning and Control, v. 1, n. 6, p. 598-607, 2000. 
PETERS, M. H.; SCHNEIDER, H.; TANG, K. Joint determination of optimal inventory and quality control policy. Management Science, v. 34, n. 8, p. 991-1004, 1988.

PORTEUS, E. L. Optimal lot sizing, process quality improvement and setup cost reduction. Operations Research, v. 34, p. 137-144, 1986.

PORTEUS, E. L. The impact of inspection delay on process and inspection lot sizing. Management Science, v. 36, n. 8, p. 999-1007, 1990.

ROSENBLATT, M. J.; LEE, H. L. Economic production cycles with imperfect production processes. IIE Transactions, v. 18, p. 48-55, 1986a.

ROSENBLATT, M. J.; LEE, H. L. A comparative study of continuous and periodic inspection policies in deteriorating production systems. IIE Transactions, p. 2-9, 1986b.

RUMMLER, G. A.; BRACHE, A. P. Melhores desempenhos das empresas: ferramentas para a melhoria da qualidade e da competitividade. São Paulo: Makron Books do Brasil Editora Ltda., 1994.

SHIH, W. Optimal inventory policies when stockouts result from defective products, internat. $J$. Production Res., v. 18, n. 6, p. 677-686, 1980.
SIPPER, D.; BULFIN JR., R. L. Production: planning, control and integration. New York: McGraw Hill, 1997.

TAPIERO, C. S.; HSU, L. F. Quality control of the M/M/1 queue. International Journal Production Research, v. 25, n. 3, p. 447-455, 1987.

TAPIERO, C. S.; HSU, L. F. Quality control of an unreliable random FMS: with Bernoulli and CSP sampling. International Journal Production Research, v. 26, n. 6, p. 1125-1135, 1988.

URBAN, T. L. Analysis of production system when run length influences product quality. International Journal of Production Research, v. 36, n. 11, p. 3085-3094, 1998.

VAN DER BIJ, H.; VAN EKERT, J. H. W. Interaction between production control and quality control. International Journal of Operations \& Production Management, v. 19, p. 674-690, 1999.

WACKER, J. G. A theoretical model of manufacturing lead times and their relationship to a manufacturing goal hierarchy. Decision Sciences, v. 27, n. 3, p. 483-517, 1996.

YANO, C. A.; LEE, H. L. Lot sizing with random yields: a review. Operations Research, v. 43, n. 2, p. 311-334, 1995.

\title{
A SYSTEM FOR CLASSIFYING AND CODIFING PAPERS RELATING PRODUCTION CONTROL AND QUALITY CONTROL
}

\begin{abstract}
This work presents a system for classifying and codifying papers (PCCS - papers classification and codification system) relating Production Control (PC) and Quality Control (QC). The classification system is based on three main characteristics. These characteristics are: the nature, the focus and the goals of the article. The codification system uses the basic idea of the Group Technology (codification of the families of parts). An hybrid structure with three digits is used to codify the characteristics aforementioned. A survey, compounded by 45 papers (we tried to perform a survey the most complete as possible) was accomplished and used to create the PCCS. The system is validated through its use on the classification and codification of the papers found on survey. The classification and codification is useful to structure the literature about $P C$ and QC and to perform a general analysis about the theme. From the analysis of these papers, using our system, a mental clarification and communication of the researches relating $P C$ and $Q C$ is obtained. After such analysis, new research areas within the relations between PC and QC can be suggested. The paper also highlights the importance in studying the relationship between PC and QC.
\end{abstract}

Key words: production control, quality control, classification and codification system, survey. 